\title{
CÓMO HACER REVISIONES BIBLIOGRÁFICAS TRADICIONALES O SISTEMÁTICAS UTILIZANDO BASES DE DATOS ACADÉMICAS
}

\section{How to do traditional or systematic bibliographic reviews using academic databases}

\author{
Lluís CODINA \\ Universidad Pompeu Fabra. Departamento de Comunicación. Barcelona. España. \\ Correspondencia: lluis.codina@upf.edu
}

Fecha de recepción: 27 de abril de 2020

Fecha de aceptación: 29 de abril de 2020

Fecha de publicación: 17 de mayo de 2020

Fecha de publicación del fascículo: 1 de junio de 2020

Conflicto de intereses: Los autores declaran no tener conflictos de intereses

Imágenes: Los autores declaran haber obtenido las imágenes con el permiso de los pacientes

Política de derechos y autoarchivo: se permite el autoarchivo de la versión post-print (SHERPA/RoMEO)

Licencia CC BY-NC-ND. Licencia Creative Commons Atribución-NoComercial-SinDerivar 4.0 Internacional

Universidad de Salamanca. Su comercialización está sujeta al permiso del editor

RESUMEN: Introducción y objetivos: Las bases de datos académicas permiten llevar a cabo revisiones bibliográficas que estén libres de sesgos y que a la vez tengan la máxima transparencia y trazabilidad.

Método: Presentamos, mediante preguntas y respuestas, las claves principales que conectan el uso de bases de datos académicas con las revisiones bibliográficas, sistemáticas o tradicionales.

Resultados: En cada apartado se presentan enlaces a entradas relacionadas donde se amplían los temas tratados para quienes deseen profundizar en ellos.

Al final se presenta una bibliografía recomendada y se destacan las dos importantes obras en las que hemos basado principalmente este y otros trabajos anteriores sobre el mismo tema.

Conclusiones: Hemos presentado, en un sistema de preguntas y respuestas, los elementos esenciales de la relación entre bases de datos académicas y revisiones bibliográficas. Para entender esta relación hemos presentado previamente las funciones de las revisiones bibliográficas y sus productos principales. También hemos discutido con cierto detalle las características de la principal dicotomía que existe en las revisiones bibliográficas, a saber, las tradicionales y las sistemáticas, con su alternativa para Ciencias Sociales y Humanidades, que no siempre trabajan sobre intervenciones o sobre resultados de investigación, que son las aproximaciones sistemáticas. 


\section{CÓMO HACER REVISIONES BIBLIOGRÁFICAS TRADICIONALES O SISTEMÁTICAS UTILIZANDO BASES DE DATOS ACADÉMICAS \\ CODINA L}

En el apartado de las bases de datos académicas hemos desarrollado un grupo de preguntas y respuestas sobre su utilización. Mostrando los componentes principales de las bases de datos académicas, así como un procedimiento basado en 6 fases, han sido mostrados igualmente.

En la mayoría de los apartados, además hemos añadido enlaces a recursos relacionados, consistentes en entradas publicadas en este mismo sitio donde se desarrollan, in extenso, algunos de tales apartados.

PALABRAS CLAVE: Documentación; revisiones bibliográficas; revisiones sistematizadas; etiquetas; navegación de entradas; bases de datos.

SUMMARY: Introduction and objectives: Academic databases allow bibliographic reviews to be carried out that are free of bias and at the same time have maximum transparency and traceability.

Method: We present, through questions and answers, the main keys that connect the use of academic databases with bibliographic, systematic or traditional reviews.

Results: In each section, links to related entries are presented where the topics discussed are expanded for those who wish to study them further.

At the end a recommended bibliography is presented and the two important works on which we have mainly based this and other previous works on the same subject are highlighted.

Conclusions: We have presented, in a question and answer system, the essential elements of the relationship between academic databases and bibliographic reviews. To understand this relationship, we have previously presented the functions of bibliographic reviews and their main products. We have also discussed in some detail the characteristics of the main dichotomy that exists in bibliographic reviews, namely traditional and systematic, with its alternative for Social Sciences and Humanities, which do not always work on interventions or on research results, which are the systematic approaches.

In the academic databases section, we have developed a group of questions and answers about their use. Showing the main components of the academic databases, as well as a procedure based on 6 phases, have also been shown.

In most of the sections, we have also added links to related resources, consisting of entries published on this same site where some of these sections are developed in extenso.

KEYWORDS: Documentation; bibliographic reviews; systematized reviews; labels; ticket navigation; databases.

\section{INTRODUCCIÓN}

La serie de preguntas y respuestas se presenta en dos partes:

- Revisiones bibliográficas tradicionales y sistemáticas, y su alternativa, las revisiones sistematizadas.

- Rol de las bases de datos y cómo usarlas en revisiones bibliográficas, del tipo que sean.

Debemos señalar que nuestro background en todo lo que seguirá son las Ciencias Sociales y las Humanidades, y aún más concretamente, los estudios de comunicación social.

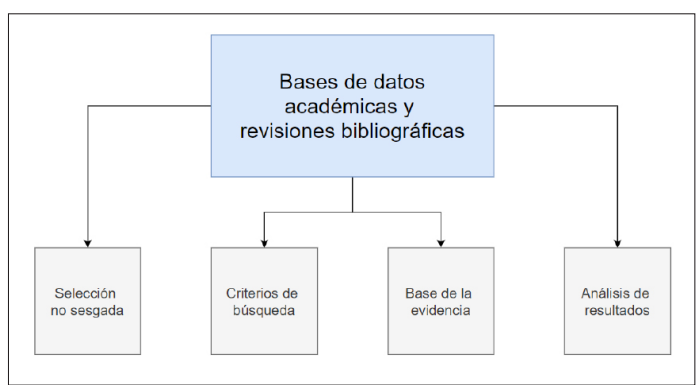

Figura 1. La conexión de las bases de datos académicas y las revisiones bibliográficas (All types of review should be systematic - Booth, Shutton, Papaioannou.2016-) 


\section{CÓMO HACER REVISIONES BIBLIOGRÁFICAS TRADICIONALES O SISTEMÁTICAS UTILIZANDO BASES DE DATOS ACADÉMICAS}

CODINA L

PRIMERA PARTE:

\section{REVISIONES BIBLIOGRÁFICAS}

¿QUÉ FUNCIONES TIENEN LAS REVISIONES BIBLIOGRÁFICAS?

Las revisiones bibliográficas (literary reviews) tienen un papel esencial en el avance de la ciencia debido al carácter acumulativo de la misma. Cumplen un amplio abanico de funciones entre las que podemos señalar las siguientes (Figura 2):

\section{Funciones de las revisiones bibliográficas}

$>$ Dan garantías a los evaluadores de que los autores conocen el ámbito de la investigación

$>$ Es la primera demostración de que un investigador sabe manejar, analizar e interpretar información científica

$>$ Evitan la repetición de investigaciones y el uso de teorías obsoletas o desacreditadas

$>$ Ayudan a detectar huecos y oportunidades de investigación

$>$ Proporcionan ideas y modelos para nuevas investigaciones

$>$ Proporcionan el marco teórico y metodológico necesario para afrontar nuevas investigaciones Aportan la base de la evidencia para estados de la cuestión y trabajos de revisión

Figura 2. Funciones de las revisiones bibliográficas (Fuente: elaboración propia).

- En artículos científicos y en memorias de solicitud de proyectos financiados proporcionan garantías a los evaluadores. Demuestran que los autores conocen el campo en el que han llevado a cabo su trabajo.

- En tesis doctorales o de máster, es la primera demostración de que los autores son capaces de analizar e interpretar críticamente trabajos científicos.

- Para los trabajos de revisión constituyen la base de la evidencia.

- En los nuevos proyectos proporcionan acceso a las teorías y conceptos que forman la corriente principal del campo en el que se pretende realizar aportaciones.

- Evitan el riesgo de repetir investigaciones o de utilizar teorías obsoletas o desacreditadas.

- Sirven para identificar huecos y oportunidades de investigación y proporcionan el marco teórico y metodológico que permite hacer aportaciones propias e incluso ser innovadores al afrontar nuevas investigaciones.

- Son la base de una serie de productos académicos, tales como los estados de la cuestión y los trabajos de revisión.

¿QUÉ PRODUCTOS OBTENEMOS DE UNA REVISIÓN BIBLIOGRÁFICA?

El resultado de una revisión bibliográfica, o literary review, puede consistir en alguno de estos productos, aunque no hay una lista cerrada (Figura 3):

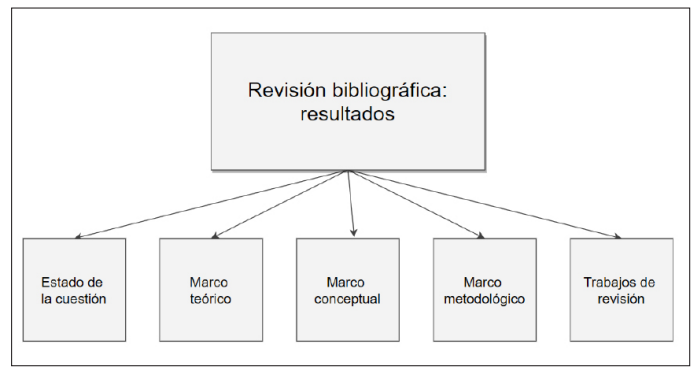

Figura 3. Posibles resultados de las revisiones bibliográficas.

- Estado de la cuestión (state of the art): una revisión de tipo global de un ámbito del conocimiento. Suele formar parte de un trabajo más amplio, como una tesis doctoral, por ejemplo.

- Marco conceptual (conceptual framework), teórico (theoretical framework) o metodológico (methological framework): presentación más o menos detallada del aparato conceptual, teórico o metodológico en el que se basa una investigación.

- Trabajo de revisión (literary review o systematic review): una investigación basada en la 


\section{CÓMO HACER REVISIONES BIBLIOGRÁFICAS TRADICIONALES O SISTEMÁTICAS UTILIZANDO BASES DE DATOS ACADÉMICAS \\ CODINA L}

evidencia publicada en resultados de investigaciones sobre una intervención o un programa determinado.

- Antecedentes teóricos o metodológicos en artículos científicos. Demuestra que los autores conocen el terreno que pisan. Sin este apartado, la mayor parte de los editores de revistas científicas rechazarán el trabajo.

\section{TrABAJOS DE REVISIÓN}

En el caso de los trabajos de revisión, la totalidad de estos consiste en una revisión bibliográfica, en especial cuando se han llevado a cabo mediante el protocolo de las revisiones sistemáticas (aunque no únicamente). En este caso, no son el complemento de un trabajo más amplio, sino que son el trabajo científico en sí mismo.

Esta naturaleza de género científico por derecho propio nació en el ámbito de la medicina, gracias a la labor de centros como la Cochrane Collaboration. Después, por la influencia de esta disciplina en las demás ciencias, ha ido siendo reconocido prácticamente en todos los ámbitos científicos.

\section{Recurso}

- Página de resultados de Science Direct de artículos con las palabras clave "literary review» o «systematic review»: https://www.sciencedirect.com/search/advanced?qs=\%22literary $\% 20$ review $\% 22 \% 20$ OR $\% 20 \% 22$ systematic $\% 20$ review $\% 22$

- Sitio web de la revista open access Systematic Reviews: https://systematicreviewsjournal. biomedcentral.com/

¿QUÉ DIFERENCIAS HAY ENTRE LAS REVISIONES BIBLIOGRÁFICAS TRADICIONALES Y SISTEMÁTICAS?

Las diferencias entre la revisión de uno u otro tipo las vamos a establecer en base a estos criterios:

- Protocolo

- Foco
- Comparación

- Base de la evidencia

- Trazabilidad

- Número de autores

- Objetivos

- Resultados

- Calidad

- Registro

- Fiabilidad

\section{Protocolo}

Las revisiones bibliográficas tradicionales no declaran protocolos específicos de desarrollo, aparte de las buenas prácticas generales aplicables a todos los trabajos académicos (ver, por ejemplo, las recomendaciones JARS de la norma APA — https:// apastyle.apa.org/jars-). Las revisiones sistemáticas, en cambio, deben declarar y hacer explícitos una serie de aspectos:

- Fuentes utilizadas y procedimiento de búsqueda utilizados.

- Criterios de inclusión y exclusión para delimitar la base de la evidencia (esto es, el grupo de documentos utilizados para el análisis).

- Esquemas de análisis utilizados para examinar de manera sistemática todos los documentos.

- Procedimientos de síntesis y de presentación de resultados.

Lo anterior, queda muy bien recogido en el llamado framework SALSA (Grant y Booth, 2009), que es el acrónimo de lo siguiente:

- Search

- AppraisaL

- Sinthesys

- Analysis

Foco

Las revisiones sistemáticas se centran en intervenciones (de salud, originalmente, pero con el tiempo se han ampliado a programas, políticas o tratamientos), a fin de comprobar su supuesta eficiencia. Por esta razón, el diseño de las revisiones 


\section{CÓMO HACER REVISIONES BIBLIOGRÁFICAS TRADICIONALES O SISTEMÁTICAS UTILIZANDO BASES DE DATOS ACADÉMICAS \\ CODINA L}

sistemáticas debe basarse en preguntas de investigación.

En cambio, uno de los usos más característicos de las revisiones tradicionales es para presentar estados de la cuestión o marcos teóricos. Por esta razón, en el diseño de una revisión tradicional, el autor se pregunta por las teorías y conceptos principales en un determinado ámbito.

\section{Qué se compara}

En las revisiones sistemáticas suelen compararse resultados de investigación sobre la eficacia de una determinada intervención o determinada metodología lo que permite establecer esquemas unificados de análisis y la posterior comparación de tales resultados bajo criterios unificados.

En las revisiones tradicionales suelen compararse las ideas, conceptos o constructos teóricos de diferentes autores y trabajos. No se acostumbra a hacer explícitos esquemas de comparación. La eficacia de esta depende de la habilidad intelectual o narrativa del autor de la revisión. No es posible saber si todos los trabajos se analizaron con los mismos criterios.

\section{Base de la evidencia}

En las revisiones sistemáticas el grupo de documentos seleccionados se considera la base de la evidencia. Esto es un concepto que no se maneja en las revisiones tradicionales. En las primeras se utilizan preferentemente artículos de revistas científicas, por ser los vectores principales de resultados de investigación, aunque no hay ningún tipo de documento excluido a priori.

En las revisiones tradicionales pueden utilizarse artículos de revistas científicas, pero también pueden utilizarse obras de pensamiento o de tipo ensayístico porque lo importante es conocer los paradigmas dominantes en una disciplina.

Se utilizan criterios bien definidos en las revisiones sistemáticas para seleccionar las investigaciones, por ejemplo, ecuaciones de búsqueda en bases de datos concretas.

En revisiones tradicionales no hay criterios explícitos. El autor se guía por su conocimiento del campo o por las obras que le han impactado más. Aunque la revisión tradicional sea de calidad, no es posible garantizar la inexistencia de sesgos en la selección de las obras consideradas.

\section{Trazabilidad}

En revisiones bibliográficas tradicionales, la trazabilidad no queda establecida, y ni siquiera es una preocupación reconocida. Los autores no explican cómo seleccionaron los trabajos que forman parte de su revisión ni qué fuentes utilizaron. La única trazabilidad es la lista de referencias, pero no es posible saber si quedaron fuera obras que debieron formar parte, o si se han incluido obras que no debieron formar parte.

Por su parte, en las revisiones sistemáticas deben señalarse de forma explícita los criterios de inclusión y exclusión que se han seguido para reunir la base de la evidencia, esto es, el grupo de trabajos sobre los que se lleva a cabo la revisión.

\section{Número de autores}

El protocolo de la institución más importante en la medicina basada en la evidencia (origen de las systematic reviews), la Cochrane Collaboration (https://es.cochrane.org/es), señala en el Cochrane Handbook for Systematic Reviews of Interventions (https://training.cochrane.org/handbook/current) que para generar revisiones sistemáticas se requieren al menos dos revisores, a fin de asegurar la consistencia en diversas fases del trabajo.

Por su parte, las revisiones tradicionales no establecen nada al respecto. Con frecuencia una revisión tradicional puede tener dos o más autores, pero también puede haber sido desarrollado por uno solo. 


\section{CÓMO HACER REVISIONES BIBLIOGRÁFICAS TRADICIONALES O SISTEMÁTICAS UTILIZANDO BASES DE DATOS ACADÉMICAS \\ CODINA L}

\section{Objetivos}

Como ya hemos señalado, en las revisiones sistemáticas se busca conocer la eficacia de una intervención o la precisión de tests de diagnóstico. En las revisiones tradicionales es frecuente que el objetivo sea identificar las teorías (o el paradigma dominante) más importantes en un ámbito.

Por supuesto, según el proyecto puede haber otros objetivos en el caso de las tradicionales, como revisar metodologías o la evolución histórica de un concepto. Pero las destinadas a establecer los paradigmas son muy características por su utilización en tesis doctorales y en memorias de proyectos de investigación.

Es evidente que en todos los casos los objetivos son valiosos. En las sistemáticas, porque ayudar a conocer de una forma fiable (ciencia basada en la evidencia) la eficacia de un tratamiento o de una intervención, es imposible de exagerar. En las tradicionales porque suelen aportar la necesaria visión holística de un ámbito de la ciencia para identificar tendencias u oportunidades de investigación, así como facilitan la comunicación interdisciplinaria.

\section{Resultados}

En las revisiones tradicionales los resultados de la revisión suelen consistir en estados de la cuestión o marcos teóricos, expresados en general como síntesis narrativas.

Las revisiones sistemáticas aportan metaanálisis respecto a los resultados cuantitativos, y meta síntesis en los resultados cualitativos. Suelen presentarse de diversas maneras, con síntesis narrativas, pero también con diagramas y tablas.

\section{Registro}

En las revisiones tradicionales no solo se admite que el autor lleve a cabo observaciones propias: subjetivas, interpretativas o críticas de los trabajos reseñados, sino que en ocasiones se reclama como un mérito de las mismas.
En las revisiones sistemáticas se espera, sobre todo, que los autores sean eficaces en lograr la síntesis lo más objetiva posible de diversos resultados de investigaciones, aunque no se excluyen ni mucho menos las interpretaciones.

\section{Calidad}

Tanto las revisiones tradicionales como las sistemáticas pueden tener una gran calidad. Las primera son enteramente dependientes de la experiencia y los conocimientos del autor, así como de las habilidades narrativas del mismo.

Las segundas, lo son mucho más dependientes de la eficacia en la selección y aplicación de los elementos del protocolo y de su tratamiento de forma rigurosa, aunque los autores también deben tener buena formación en su campo.

\section{Fiabilidad}

Aunque una revisión tradicional puede tener una gran calidad, como ya hemos señalado, no siempre es posible determinar su grado de fiabilidad. Al no seguir protocolos, o no hacerlos explícitos, la fiabilidad no queda ni negada ni afirmada.

En las revisiones sistemáticas es justamente la fiabilidad lo que queda mejor establecida. Al seguir un protocolo que se hace explícito, su fiabilidad es equivalente a la de un género científico, y así es considerado en muchas disciplinas, especialmente en las ciencias de la salud.

\section{Recurso}

- Revisiones sistematizadas y cómo llevarlas a cabo con garantías: systematic reviews y SALSA Framework: https://www.lluiscodina.com/revision-sistematica-salsa-framework/

\section{¿HAY VARIOS TIPOS DE REVISIONES BIBLIOGRÁFICAS?}

Nosotros utilizamos la dicotomía entre las revisiones tradicionales y las sistemáticas porque nos interesa poner la línea divisoria entre las que 


\section{CÓMO HACER REVISIONES BIBLIOGRÁFICAS TRADICIONALES O SISTEMÁTICAS UTILIZANDO BASES DE DATOS ACADÉMICAS \\ CODINA L}

utilizan y hace explícitos, o no, los protocolos para llevarlas a cabo.

Pero atendiendo a otros criterios, como los objetivos, los métodos, el grado de sistematicidad, los apartados en los que se centra el análisis, etc., podemos tener otras tipologías. En concreto, el épico (si se nos permite esta expresión) artículo de Grant y Booth de 2009 establece hasta catorce tipos con sus definiciones correspondientes.

Reproducimos a continuación la tipología de Grant y Booths (2009) con las descripciones literales tomadas de su artículo:

- Critical review: Aims to demonstrate writer has extensively researched literature and critically evaluated its quality. Goes beyond mere description to include degree of analysis and conceptual innovation. Typically results in hypothesis or model.

- Literature review: Generic term: published materials that provide examination of recent or current literature. Can cover wide range of subjects at various levels of completeness and comprehensiveness. May include research findings.

- Mapping review/Systematic map: Map out and categorize existing literature from which to commission further reviews and/or primary research by identifying gaps in research literature.

- Meta-analysis: Technique that statistically combines the results of quantitative studies to provide a more precise effect of the results.

- Mixed studies review/mixed methods review: Refers to any combination of methods where one significant component is a literature review (usually systematic). Within a review context it refers to a combination of review approaches for example combining quantitative with qualitative research or outcome with process studies.

- Overview: Generic term: summary of the [medical] literature that attempts to survey the literature and describe its characteristics.

- Qualitative systematic review/qualitative evidence synthesis: Method for integrating or comparing the findings from qualitative studies. It looks for 'themes' or 'constructs' that lie in or across individual qualitative studies

- Rapid review: Assessment of what is already known about a policy or practice issue, by using systematic review methods to search and critically appraise existing research

- Scoping review: Preliminary assessment of potential size and scope of available research literature. Aims to identify nature and extent of research evidence (usually including ongoing research).

- State-of-the-art review: Tend to address more current matters in contrast to other combined retrospective and current approaches. May offer new perspectives on issue or point out area for further research.

- Systematic review: Seeks to systematically search for, appraise and synthesis research evidence, often adhering to guidelines on the conduct of a review.

- Systematic search and review: Combines strengths of critical review with a comprehensive search process. Typically addresses broad questions to produce 'best evidence synthesis'

- Systematized review: Attempt to include elements of systematic review process while stopping short of systematic review. Typically conducted as postgraduate student assignment.

- Umbrella review: Specifically refers to review compiling evidence from multiple reviews into one accessible and usable document. Focuses on broad condition or problem for which there are competing interventions and highlights reviews that address these interventions and their results.

Retenemos de esta tipología, que hay hasta tres tipos de revisión que incluyen el término systematic o la derivación systematized en su denominación. 


\section{CÓMO HACER REVISIONES BIBLIOGRÁFICAS TRADICIONALES O SISTEMÁTICAS UTILIZANDO BASES DE DATOS ACADÉMICAS \\ CODINA L}

¿HAY Alguna ALternativa A LA Dicotomía TRADICIONAL VS SISTEMÁTICA?

Tesis

Hemos visto que las revisiones sistemáticas aportan beneficios como el rigor, la trazabilidad y la transparencia a una revisión bibliográfica, pero su foco es relativamente limitado, ya que se centran en investigaciones, tests o metodologías.

\section{Antítesis}

También hemos visto que, aunque las revisiones tradicionales no suelen demostrar su fiabilidad, tienen un foco mucho más amplio que es necesario preservar. Este foco es imprescindible en determinados trabajos académicos, notablemente en tesis doctorales o en memorias de solicitud de proyectos de investigación, por mencionar únicamente dos casos.
SÍNTESIS: REVISIONES CON APROXIMACIONES SISTEMÁTICAS

La posible (y seguramente deseable) solución consiste en utilizar aproximaciones sistematizadas en todo tipo de revisiones, independientemente de sus objetivos, foco, etc. Parece difícil objetar sobre esto, porque aseguran la validez y la fiabilidad. Con ello, disparan la calidad académico-científica de las revisiones. Veamos que señalan al respecto Booth, Sutton y Papaioannou, (2016b):

All types of review should be systematic. Being systematic helps you to reduce the likelihood of bias and is a way of ensuring that you identify a comprehensive body of knowledge on your chosen subject. You will encounter many definitions for literature reviews, but you will find that the word 'systematic' often features as a critical element within the description of a literature review.

\begin{tabular}{|c|c|c|c|c|c|}
\hline \multirow[b]{2}{*}{ Label } & \multirow[b]{2}{*}{ Description } & \multicolumn{4}{|l|}{ Methods used (SALSA) } \\
\hline & & Search & Appraisal & Synthesis & Analysis \\
\hline Critical review & $\begin{array}{l}\text { Aims to demonstrate writer has extensively } \\
\text { researched literature and critically evaluated its } \\
\text { quality. Coes beyond mere description to include } \\
\text { degree of analysis and conceptual innovation. } \\
\text { Typically results in hypothesis or model }\end{array}$ & $\begin{array}{l}\text { Seeks to identify } \\
\text { most significant items } \\
\text { in the field }\end{array}$ & $\begin{array}{l}\text { No formal quality } \\
\text { assessment. Attempts } \\
\text { to evaluate according } \\
\text { to contribution }\end{array}$ & $\begin{array}{l}\text { Typically narrative, } \\
\text { perhaps conceptual } \\
\text { or chronological }\end{array}$ & $\begin{array}{l}\text { Significant component: seeks to } \\
\text { identify conceptual contribution } \\
\text { to embody existing or derive } \\
\text { new theory }\end{array}$ \\
\hline $\begin{array}{l}\text { Mapping review/ } \\
\text { systematic map }\end{array}$ & $\begin{array}{l}\text { Map out and categorize existing literature } \\
\text { from which to commission further reviews } \\
\text { and/or primary research by identifying } \\
\text { gaps in research literature }\end{array}$ & $\begin{array}{l}\text { Completeness of } \\
\text { searching determined } \\
\text { by time/scope } \\
\text { constraints }\end{array}$ & $\begin{array}{l}\text { No formal quality } \\
\text { assessment }\end{array}$ & $\begin{array}{l}\text { May be graphical } \\
\text { and tabular }\end{array}$ & $\begin{array}{l}\text { Characterizes quantity and } \\
\text { quality of literature, perhaps by } \\
\text { study design and other key } \\
\text { features. May identify need for } \\
\text { primary or secondary research }\end{array}$ \\
\hline
\end{tabular}

Figura 4. Tipos de revisiones con el framework SALSA (Grant y Booth, 2009 en: https://onlinelibrary.wiley.com/doi/ epdf/10.1111/j.1471-1842.2009.00848.x). 


\section{CÓMO HACER REVISIONES BIBLIOGRÁFICAS TRADICIONALES O SISTEMÁTICAS UTILIZANDO BASES DE DATOS ACADÉMICAS \\ CODINA L}

La sistematización no solo ayuda a los lectores de la revisión, ayuda también a los autores de la revisión, porque al evitar que caigan en sesgos, mejorará su conocimiento del ámbito que están revisando. Como consecuencia, las revisiones con aproximaciones sistemáticas generan autores más sabios.

Esto, a su vez, generará una mejor ciencia en general. Es fácil ver la relación: si el autor de una tesis doctoral, por citar uno solo de los escenarios, desarrolla su marco teórico y metodológico sin caer en sesgos, su investigación será mucho mejor. Si esto se puede escalar a todos los casos en los que un revisor hace su trabajo, el beneficio para el progreso de la ciencia será indudable.

¿Qué CaraCteriza a las aproximaciones SISTEMÁTICAS?

La sistematización de una revisión se consigue aplicando un protocolo que aporte transparencia, rigor y trazabilidad a las diferentes fases de desarrollo de la revisión.

Las principales características de las revisiones bibliográficas que se adhieren a una aproximación sistemática son los siguientes:

- Establece la base de la evidencia o banco de documentos sobre principios de transparencia y trazabilidad.

- Identifica y selecciona los documentos mediante fuentes fiables, como bases de datos académicas, y con criterios explícitos de inclusión y exclusión.

- Presenta esquemas explícitos de análisis de los documentos.

- Presenta los resultados mediante diferentes estrategias de síntesis, como tablas, diagramas y, por supuesto, síntesis narrativas (como en las revisiones tradicionales).

\section{Recursos}

- Revisiones sistematizadas para trabajos académicos. Conceptos, fases y bibliografía: https:// www.lluiscodina.com/revisiones-sistematizadas-fundamentos/

- Revisiones sistematizadas para trabajos académicos. Fases de Búsqueda y Evaluación: https:// www.lluiscodina.com/revisiones-sistematizadas-busqueda-evaluacion/

- Revisiones sistematizadas para trabajos académicos. Análisis y Síntesis: https://www. lluiscodina.com/revisiones-sistematizadasanalisis-sintesis/

¿EN QUÉ CONSISTE LA BASE DE LA EVIDENCIA EN UN TRABAJO DE REVISIÓN?

En un trabajo de investigación, la base de la evidencia son los datos que van a ser objeto de análisis. Pueden ser entrevistas, datos de una encuesta o de un focus group, de un trabajo de campo, etc.

Por su parte, en revisiones bibliográficas, la base de la evidencia la constituyen los documentos que se van a analizar. Esto es, las referencias con las que vamos a desarrollar el estado de la cuestión, el marco teórico o el trabajo de revisión.

\section{EviTAR SESGOS}

Como en cualquier otro género de investigación, necesitamos que la base de la evidencia esté desprovista, en lo humanamente posible, de sesgos. El instrumento más eficaz para evitar tales sesgos son los sistemas de información académica, entre los que destacan las bases de datos académicas. No obstante, no son, ni mucho menos, las únicas fuentes posibles, aunque como explicaremos más adelante, se encuentran entre las más fiables.

Lo que es importante es que la base de la evidencia no se haya obtenido (solamente) por decantación, esto es, por el conocimiento previo del autor, sino que haya sido obtenida siguiendo unos criterios bien establecidos de inclusión y exclusión de trabajos. También mediante selección e identificación de las fuentes utilizadas. 


\section{CÓMO HACER REVISIONES BIBLIOGRÁFICAS TRADICIONALES O SISTEMÁTICAS UTILIZANDO BASES DE DATOS ACADÉMICAS \\ CODINA L}

Como ya hemos señalado, un término alternativo al de base de la evidencia, es el de banco de documentos. Entonces, se entiende que el banco de documentos está formado por los trabajos que serán objeto de la revisión.

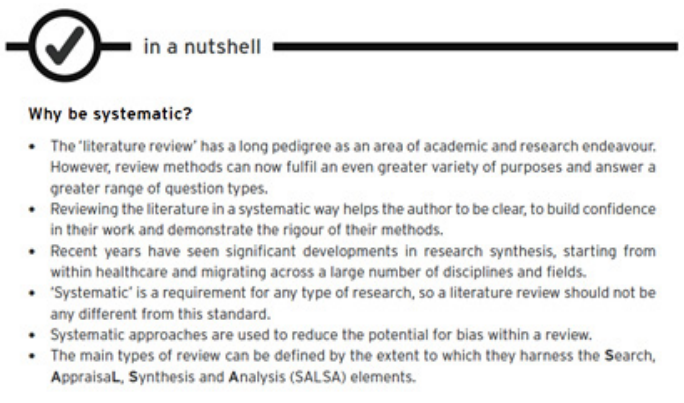

Figura 5. Fuente: Booth, Andrew; Sutton, Anthea; Papaioannou, Diana (2016b).

\section{¿QUÉ COMPONENTES O FASES TIENE UNA REVISIÓN} BIBLIOGRÁFICA SISTEMATIZADA?

A partir de la propuesta, varias veces mencionadas de Booth y sus colegas, se suele utilizar el llamado framework SALSA para identificar las cuatro fases o componentes de una revisión bibliográfica:

- Búsqueda: en esta fase se establecen los criterios por los que se buscarán o seleccionarán los trabajos que formarán parte de la base de la evidencia o el banco de documentos.

- Evaluación: consiste en los procedimientos por los cuales se examinarán los trabajos para determinar si son adecuados para formar parte de la base de la evidencia.

- Análisis: consiste en el diseño del esquema que permitirá analizar de forma sistemática los documentos que forman la base de la evidencia.

- Síntesis: consiste en las diferentes formas en las que se presentarán los resultados de los análisis de una forma comprensiva.

\section{SEGUNDA PARTE: LAS BASES DE DATOS ACADÉMICAS}

\section{¿QUÉ FUENTES PODEMOS UTILIZAR?}

Las fuentes concretas para utilizar dependen, en cada caso, del proyecto. No obstante, se suele privilegiar el uso de artículos de revista. Esto último, en especial si queremos darle los máximos rasgos de sistematicidad posible, cosa que, en teoría, como ya hemos discutido, siempre es deseable. Esto nos lleva a las bases de datos académicas.

No obstante, cualquier formato académico es susceptible de formar parte de una revisión bibliográfica. Por tanto, además de artículos científicos, podemos utilizar informes, comunicaciones, libros, etc. En cada caso, en especial en revisiones de las que queramos reclamar que se han llevado a cabo con aproximaciones sistemáticas, convendrá justificar el uso de las fuentes y su tipología.

\section{¿CUÁl ES LA FUNCIÓN DE LAS BASES DE DATOS ACADÉMICAS?}

Si hemos de apoyar la totalidad o el grueso de nuestra revisión en artículos de revistas científicas (cosa más que recomendable) el corolario inevitable es utilizar bases de datos académicas. La razón es que resulta virtualmente imposible explorar de forma eficiente la información publicada en revistas científicas en la mayoría de los ámbitos sin utilizar bases de datos.

En todo caso, sin el uso de bases de datos es imposible asegurarnos de que no hemos incurrido en sesgos en la selección de los trabajos en los que basaremos nuestra revisión, porque entonces dependeremos de nuestra memoria, nuestros gustos y afinidades, los trabajos que conocemos previamente, etc.

\section{¿CuÁles SON las PRINCIPAles bases de DATOS EN COMUNICACIÓN SOCIAL?}

La respuesta rápida es que, para la mayoría de los proyectos, se puede utilizar al menos Scopus 


\section{CÓMO HACER REVISIONES BIBLIOGRÁFICAS TRADICIONALES O SISTEMÁTICAS UTILIZANDO BASES DE DATOS ACADÉMICAS \\ CODINA L}

y Web of Science (WoS). De hecho, el estatus de estas dos bases de datos es tal que, en caso de no utilizarlas, habría que justificar la razón.

Por supuesto, además, podemos necesitar otras bases de datos dependiendo de las características de cada proyecto. En concreto, para revisiones destinadas a trabajos académicos que se van a presentar en universidades europeas puede ser muy recomendable añadir ERIH Plus (https:// www.lluiscodina.com/erih-plus/) así como Dialnet (https://www.lluiscodina.com/dialnet-plus-basede-datos/) en el caso de España.

La razón es muy simple: es importante en cada caso que los autores de trabajos académicos demuestren conocer la ciencia de su entorno. Para ser aún más pragmático: un doctorando puede verse en un aprieto si desconoce la ciencia que se hace en la universidad donde va a presentar su tesis.

No obstante, las fuentes que se deben utilizar en una revisión bibliográfica es algo que depende en cada caso de las características intrínsecas de cada investigación o de cada proyecto. Forma parte del diseño de la revisión justificar el uso de una clase $\mathrm{u}$ otra de fuentes.

En el caso de comunicación social, puede utilizarse una base de datos especializada como Communication Source (Ebsco, https://www.lluiscodina.com/communication-source/) o plataformas editoriales como las de Taylor and Francis (https:// www.lluiscodina.com/taylor-and-francis-basedatos-multisciplinaria/) o Sage (https://www.lluiscodina.com/sage-journals-base-de-datos/) por sus influyentes colecciones de revistas dedicadas a Ciencias Sociales y Humanidades, así como a comunicación social.

\section{OTRAS FUENTES}

Para aumentar el grado de cobertura, puede ser conveniente ampliar las fuentes. En tal caso, una potente fuente adicional a las que podemos acudir es Google Scholar (https://www.lluiscodina.com/ google-academico-la-ciencia-real/). En tal caso hay que tener presente que este buscador incluye toda clase de materiales académicos, no solamente artículos de revistas científicas.

También hay que tener en cuenta que esta clase de buscadores carece de un sistema de búsqueda avanzada tan completo como las bases de datos académicas señaladas, lo que dificulta la generación de ecuaciones de búsqueda. Esto último, puede complicar la fiabilidad de los resultados, algo aún peor en otros buscadores académicos como Microsoft Academic (https://www.lluiscodina. com/microsoft-academic-2019/), pese a su enorme calidad en otros aspectos.

Recursos: el concepto de grupo óptimo

- Cómo determinar el grupo óptimo de bases de datos en comunicación social: https://repositori.upf.edu/handle/10230/35705?localeattribute $=\mathrm{es}$

Recursos: análisis de las principales bases de datos académicas

- Scopus: https://www.lluiscodina.com/scopusanalisis-guia-utilizacion/

- Web of Science: https://www.lluiscodina.com/ web-of-science-wos-guia-avanzada-1/

- Dialnet Plus: https://www.lluiscodina.com/ dialnet-plus-base-de-datos/

- Índices CSIC: https://www.lluiscodina.com/ indices-csic-base-datos-academica/

- Communication \& Humanities Source: https:// www.lluiscodina.com/communication-source/

- Google Scholar: https://www.lluiscodina.com/ google-academico-la-ciencia-real/

- Microsoft Academic: https://www.lluiscodina. com/microsoft-academic-2019/

\section{¿QUÉ COMPONENTES TIENE UNA BASE DE DATOS ACADÉMICA?}

Como sea, para poder explotar la máxima potencialidad de una base de datos académica, conviene considerar que, al menos las más completas, tienen cuatro módulos principales: 


\section{CÓMO HACER REVISIONES BIBLIOGRÁFICAS TRADICIONALES O SISTEMÁTICAS UTILIZANDO BASES DE DATOS ACADÉMICAS \\ CODINA L}

- Formulario de consulta: permite expresar la necesidad de información del investigador, en general con la posibilidad de combinar varios conceptos, incluyendo variaciones y sinónimos de los mismos.

- Página de resultados: presenta los resultados en un módulo que contiene una batería enorme de funcionalidades, cuyo significado es muy importante conocer con detalle. Son de especial importancia los filtros, las formas de ordenación y las opciones de exportación.

- Registro: es una de las partes peor interpretadas por los investigadores y es peligroso, porque en los registros se encuentran algunos de los mejores recursos de las bases de datos. En concreto, aquí podemos encontrar artículos relacionados, así como métricas y altmétricas de cada artículo, junto a otras funciones, como configurar alerta de artículo.

- Módulo(s) de análisis: es otro de los módulos menos atendidos, y sin embargo puede ser uno de los más importantes. En concreto, podemos encontrar aquí análisis consistentes en distribuciones por años, autores, ámbitos de estudio, citaciones, etc.

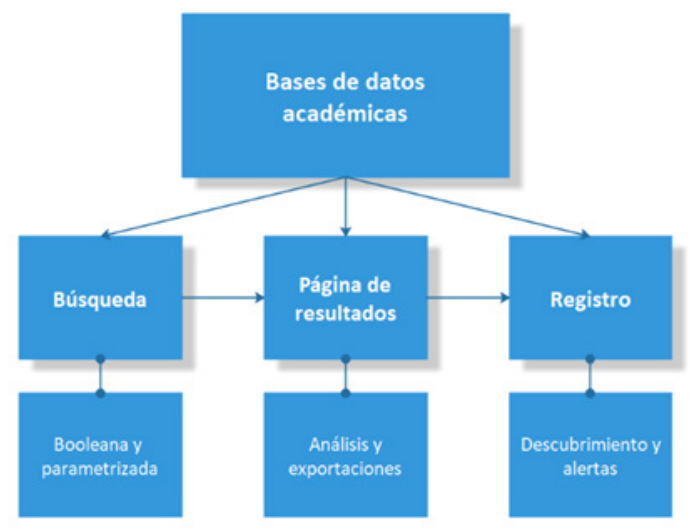

Figura 6. Componentes de una base de datos académica.

\section{Recursos}

- Formulario de consulta: https://www.lluiscodina.com/busqueda-avanzada-bases-de-datosacademicas/

- Página de resultados: https://www.lluiscodina. com/pattern-pagina-de-resultados/

- Registro: https://www.lluiscodina.com/registro-base-de-datos-academica/

- Análisis: https://www.lluiscodina.com/basesde-datos-academicas-analisis-utilidades/

¿QUÉ COMPONENTES TIENE UN FORMULARIO DE BÚSQUEDA AVANZADA?

Los principales apartados son los siguientes:

- Búsqueda booleana: permite expresar con total precisión los temas de la investigación o los temas de la revisión. En particular permite combinar dos o más conceptos, de manera que los documentos recuperados sean de alta precisión.

- Búsqueda parametrizada: permite centrar la búsqueda en campos concretos de cada documento, como por ejemplo en el título o el resumen, lo que incrementa de modo notable la precisión, evitando así al máximo posible, los falsos positivos.

- Filtros adicionales: casi todas las bases de datos, además de los anteriores, permiten añadir otros requerimientos, como rangos de fechas, tipo de documentos, idiomas, etc.

\section{Recursos}

- Búsqueda avanzada: https://www.lluiscodina. com/bases-datos-academicas-busqueda/

- Operadores booleanos: https://www.lluiscodina. com/ecuaciones-de-busqueda-bases-datosoperadores-booleanos/

- Operadores parametrizados: https://www.lluiscodina.com/ecuaciones-de-busqueda-operadores-de-proximidad/ 


\section{CÓMO HACER REVISIONES BIBLIOGRÁFICAS TRADICIONALES O SISTEMÁTICAS UTILIZANDO BASES DE DATOS ACADÉMICAS \\ CODINA L}

¿EN QUÉ CONSISTE EL PROCEDIMIENTO DE LAS 6 FASES PARA USAR BASES DE DATOS ACADÉMICAS?

Una forma de utilizar las bases de datos académicas es utilizando la propuesta basada en 6 pasos o fases que presentamos a continuación de manera sucinta:

1. Preparación, o fase de ideación y provisión de palabras clave. Probablemente, la más importante de las 6 fases. Sin una buena resolución de esta fase, todas las demás pueden conducir a una simple pérdida de tiempo.

2. Búsqueda, preferentemente utilizando un formulario de búsqueda avanzada a fin de poder representar con precisión nuestra necesidad de información.

3. Página de resultados, donde procedemos a un primer testeo de resultados, evaluando la relevancia de estos en base al título. En esta o en las dos fases siguientes podemos obtener el documento completo, si es el caso.

4. Metadatos o Registro, donde determinamos con mayor precisión la relevancia de cada resultado en base al resumen. También obtendremos otros datos y podremos activar funciones de descubrimiento.

5. Exportaciones, operación que llevamos a cabo con las referencias más relevantes y que necesitaremos poder recuperar en el futuro en cualquier momento. La exportación se realiza a un gestor de referencias como Mendeley o Zotero.

6. Alertas, mediante las cuales aseguramos que, en el futuro, estaremos conveniente informados de las novedades sin necesidad de repetir las mismas búsquedas. Dejaremos a un bot encargado de tales tareas.

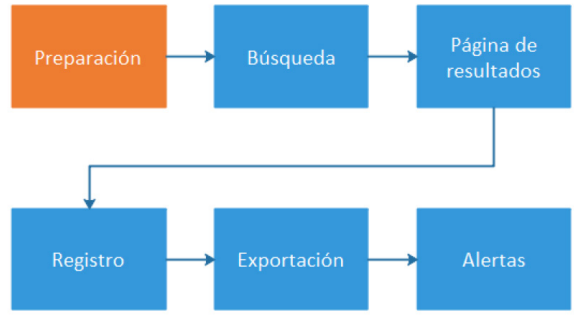

Figura 7. Procedimiento de las 6 fases para usar bases de datos académicas.

Recursos

- Uso de Scopus con el método de las 6 fases: https://www.lluiscodina.com/utilizar-bases-dedatos-academicas/

- Uso de Web of Science con el método de las 6 fases: https://www.lluiscodina.com/6-fasesweb-of-science/

- Configuración de servicios de alerta en bases de datos académicas: https://www.lluiscodina. com/ecuaciones-de-busqueda-servicios-dealerta-scholar-scopus/

¿QUÉ APORTAN LOS MÓDULOS DE ANÁLISIS DE LAS BASES DE DATOS ACADÉMICAS?

Una vez efectuada una búsqueda, las mejores bases de datos académicas proporcionan análisis de estas en base a diferentes distribuciones de datos. Como la mayoría de las distribuciones se basan en categorías, estas distribuciones se basan en análisis de frecuencias de estas, como temas, países, años, etc.

Además, proporcionan también análisis basados en citaciones, en este caso de carácter cuantitativo, notablemente número de citas y ordenaciones y rankings a partir de las mismas. 


\section{CÓMO HACER REVISIONES BIBLIOGRÁFICAS TRADICIONALES O SISTEMÁTICAS UTILIZANDO BASES DE DATOS ACADÉMICAS \\ CODINA L}

\section{Recursos}

- Análisis de resultados con bases de datos académicas: https://www.lluiscodina.com/analisis-deresultados-bases-de-datos/

- Web of Science, módulos de análisis: https:// www.lluiscodina.com/web-of-science-caracterizacion-y-guia-de-uso-avanzado-\%c2\%b72-herramientas-de-analisis/

- Scopus, módulos de análisis: https://www.lluiscodina.com/scopus-2-registro-utilidades/

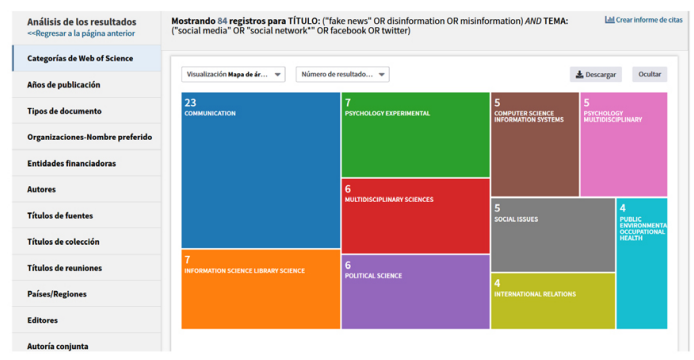

Figura 8. Análisis de resultados con bases de datos académicas.

\section{LAS BASES CONCEPTUALES}

En la bibliografía final se encontrarán las referencias en las que hemos basado esta presentación (incluyendo alguna autocitación), sin embargo, la filosofía principal de este trabajo es deudora de estas dos obras seminales, cuya importancia en el desarrollo de las revisiones sistemáticas en disciplinas distintas de la salud es imposible de exagerar:

Booth, Andrew; Sutton, Anthea; Papaioannou, Diana (2016). Systematic Approaches to a Successful Literature Review. London: Sage, 2016. [Chapter 2: Taking a Systematic Approach to Your Literature Review]

Grant, Maria J.; Booth, Andrew. (2009). A typology of reviews: an analysis of 14 review types and associated methodologies. Health Information and Libraries Journal, 26, pp.91-108.

https://onlinelibrary.wiley.com/doi/ epdf/10.1111/j.1471-1842.2009.00848.x
Bonus

Referenciamos la última edición de las normas APA porque entre los tipos de artículos científicos incluye los trabajos de revisión o metaanálisis:

American Psychological Association (2020). Chap. 3. Journal Article Reporting Standards. Publication manual of the American Psychological Association (7th ed.). https://apastyle.apa.org/jars

A propósito de las APA, nos ha parecido digno de mención que otorgan la misma denominación: metaanálisis a las síntesis de investigaciones cualitativas y cuantitativas.

En otras fuentes se suele utilizar, en cambio, el término meta síntesis en el caso de la síntesis de resultados cualitativos, y metaanálisis, cuando son cuantitativos.

\section{CONCLUSIONES}

Hemos presentado, en un sistema de preguntas y respuestas, los elementos esenciales de la relación entre bases de datos académicas y revisiones bibliográficas. Para entender esta relación hemos presentado previamente las funciones de las revisiones bibliográficas y sus productos principales.

También hemos discutido con cierto detalle las características de la principal dicotomía que existe en las revisiones bibliográficas, a saber, las tradicionales y las sistemáticas, con su alternativa para Ciencias Sociales y Humanidades, que no siempre trabajan sobre intervenciones o sobre resultados de investigación, que son las aproximaciones sistemáticas.

En el apartado de las bases de datos académicas hemos desarrollado un grupo de preguntas y respuestas sobre su utilización. Mostrando los componentes principales de las bases de datos académicas, así como un procedimiento basado en 6 fases, han sido mostrados igualmente.

En la mayoría de los apartados, además hemos añadido enlaces a recursos relacionados, consistentes en entradas publicadas en este mismo sitio donde se desarrollan, in extenso, algunos de tales apartados. 


\section{CÓMO HACER REVISIONES BIBLIOGRÁFICAS TRADICIONALES O SISTEMÁTICAS UTILIZANDO BASES DE DATOS ACADÉMICAS \\ CODINA L}

\section{DECLARACIÓN DE INTERESES}

Este artículo procede de la entrada de blog «Cómo hacer revisiones bibliográficas tradicionales o sistemáticas utilizando bases de datos académicas» del autor publicado el 27 de abril de 2020 disponible en https://www.lluiscodina.com/ revisiones-bibliograficas-bases-de-datos/.

\section{REFERENCIAS}

American Psychological Association. Qualitative Meta-Analysis Article Reporting Standards. 2020.

American Psychological Association. Chap. 3 Journal Article Reporting Standards. Publication manual of the American Psychological Association (7th ed.). 2020.

Barnett-Page E, Thomas J. Methods for the synthesis of qualitative research: a critical review. BMC Med Res Methodol. Aug. 2009.

Bloomberg LD, Volpe M. Literature Review and Theoretical/Conceptual Framework. Completing Your Qualitative Dissertation: A Road Map From Beginning to End. 3r ed. London: Sage Publishing. 2016.

Boland, A, Cherry MG, Dickson R. Doing a Systematic Review: A Student's Guide. London: Sage. 2014

Booth, Andrew. Searching for qualitative research for inclusion in systematic reviews: a structured methodological review. Systematic Reviews. 2016;5,74. https://doi.org/10.1186/s13643-0160249-x

Booth A, Sutton A, Papaioannou D. Chapter 2: Taking a Systematic Approach to Your Literature Review. Systematic Approaches to a Successful Literature Review. London: Sage. 2016

Booth A, Sutton A, Papaioannou D. Systematic Approaches to a Successful Literature Review. London: Sage. 2016.

Codina L. Revisiones bibliográficas sistematizadas: procedimientos generales y Framework para ciencias humanas y sociales. 2018. https://repositori. upf.edu/handle/10230/34497
Dixon-Woods M, Cavers D, Agarwal S et al. (2006). Conducting a critical interpretive synthesis of the literature on access to healthcare by vulnerable groups. BMC Med Res Methodol. 2006;6, 35. https://doi.org/10.1186/1471-2288-6-35

Fink A. Conducting Research Literature Reviews: From the Internet to Paper: London: Sage. 2014

Gough D, Oliver S, Thomas J. An Introduction to Systematics Reviews. London: Sage. 2012

Grant MJ, Booth A. A typology of reviews: an analysis of 14 review types and associated methodologies. Health Information and Libraries Journal. 2009, 26, pp.91-108. https://onlinelibrary.wiley.com/doi/ epdf/10.1111/j.1471-1842.2009.00848.x

Hart Ch. Doing a Literature Review: Releasing the Social Science Research Imagination. London: Sage, 2008.

Higgins, J.; Thomas, J. Cochrane Handbook for Systematic Reviews of Interventions. https://training. cochrane.org/handbook/current

Jesson JK, Matheson L, Lacey FM. Doing your Literature Review: Traditional and Systematic Techniques. London: Sage. 2011

Machi LA; McEvoy BT. The Literature Review: Six Steps to Success. Thousand Oaks, California: Corwin. 2012

Noyes, Jane; Booth, Andrew; Cargo, Margaret, et al. (2019). Chapter 21: Qualitative evidence. Higgins, J.; Thomas, J. Cochrane Handbook for Systematic Reviews of Interventions. https://training. cochrane.org/handbook/current

Pan ML. Preparing Literature Reviews: Qualitative and Quantitative Approaches. London: Routledge. 2017.

Petticrew M; Roberts H. Systematic Reviews in the Social Sciences: A Practical Guide. Oxford: Blackwell. 2006.

Popay J et. al. Guidance on the Conduct of Narrative Synthesis in Systematic Reviews: A product from the ESRC Methods Programme. 2006.

Ridley D. The Literature Review: A Step-by-Step Guide for Students. London: Sage. 2012. 